\title{
Asymmetrical modulation of time perception by increase versus decrease in coherence of motion
}

\author{
Hakan Karșılar ${ }^{1}$ • Fuat Balcı ${ }^{1}$ \\ Published online: 15 August 2016 \\ (C) The Psychonomic Society, Inc. 2016
}

\begin{abstract}
Stimulus properties are known to affect duration judgments. In this study, we tested the effect of motion coherence levels in randomly moving dots on the perceived duration of these stimuli. In Experiments 1 and 2 we tested participants on a temporal reproduction task, using stimuli with varying degrees of motion coherence as the to-be-timed stimuli. Our results in both experiments showed that increasing motion coherence from the encoded (i.e. the first) to the reproduced (i.e. the second) stimulus leads to longer reproduction times. These effects were primarily additive in nature, and their magnitude increased with the difference between the coherence levels in the encoding versus reproduction (decoding) phases. This effect was not mirrored when there was a decrease in motion coherence. Experiment 3 tested if the differential number of exploratory saccadic eye-movements during encoding and reproduction predicted these effects. The behavioral findings of Experiment 1 and 2 were replicated in the third experiment, and the change in the number of eye movements from encoding to reproduction predicted the reproduction time when there was an increase in motion coherence. These results are explained by the effect of attention on the latency to initiate temporal integration that is only manifested when there is an increase in the level of motion coherence.
\end{abstract}

Electronic supplementary material The online version of this article (doi:10.3758/s13414-016-1181-9) contains supplementary material, which is available to authorized users.

Fuat Balc1

fbalci@ku.edu.tr

1 Department of Psychology, Koç University, Rumelifeneri Yolu, Sariyer, Istanbul 34450, Turkey
Keywords Time perception $\cdot$ Signal-to-noise ratio $\cdot$ Random dot motion $\cdot$ Temporal reproduction $\cdot$ Saccadic eye movements

Accurate timing ability is essential to many daily cognitive tasks (Allan, 1979; Buhusi \& Meck, 2005). However, a constantly growing body of literature shows that the subjective experience of time is highly susceptible to subtle changes in nontemporal stimulus properties (Eagleman, 2008; Eagleman \& Pariyadath, 2009). For instance, Xuan, Zhang, He, and Chen (2007) have shown that an increase in the magnitude of various properties of the to-be-timed stimulus (e.g., its size, luminance, numeric value) lead to longer temporal judgments (also see Berglund, Berglund, Ekman, \& Frankenhaeuser, 1969; Rammsayer \& Verner, 2014, for similar effects in other domains). These so called temporal illusions are thought to be mediated by different arousal and attentional levels induced by the respective stimuli, with implications regarding the neural mechanisms that underlie time perception (see Merchant, Harrington, \& Meck, 2013, for a review).

Although the majority of the aforementioned studies systematically tested the effect of the magnitude of stimulus properties on the subjective experience of duration, to our knowledge, no study has investigated the effect of motion coherence levels (SNR; signal-to-noise ratio of motion direction) on time perception. To this end, by employing a temporal reproduction paradigm and using random dot motion (RDM) stimuli as the to-be-timed stimulus (see Gold \& Shadlen, 2001), this study aimed to elucidate the effect of the level of coherent motion on the perception of supra-second intervals. To further explicate any potential relationship between the perceived durations and the differential number of eye movements elicited by various levels of motion coherence from encoding to reproduction (e.g., Burr, Ross, Binda \& Morrone, 2010; Penney et al. 2016; Suzuki \& Yamazaki, 2010), we conducted an additional 
experiment where the eye movements of participants were recorded while being tested in identical task conditions.

The typical result from research on the effect of motion on perceived duration suggests that moving stimuli are perceived to last longer compared to stationary ones (Brown, 1995), and this distortion (i.e., dilation) in the perception of time intervals increases as a function of the speed of movement (e.g., Beckmann \& Young, 2009; Kaneko \& Murakami, 2009; Tomassini, Gori, Burr, Sandini, \& Morrone, 2011). For instance, Matthews (2011) has shown that a constantly moving stimulus is perceived to last longer than a decelerating one, which in turn seems to last longer than an accelerating stimulus. Kanai, Paffen, Hogendoorn, and Verstraten (2006), on the other hand, have used flickering stimuli to show that temporal frequency is more critical to the lengthening of the perceived duration than speed or spatial frequency (but see Kaneko \& Murakami, 2009), whereas coherence was found to have no effect at all on the perceived durations. However, Yamamoto and Miura (2016) have recently shown that, depending on stimulus configurations, the perceived speed and the coherence of motion in line segments affects perceived time, further demonstrating the role of motion processing on interval timing. Thus, although the debate as to how motion is related to time perception is not settled, no study so far has used RDM (or related) stimuli to formally define and systematically manipulate the amount of coherent motion (i.e., SNR) in a timed stimulus while simultaneously keeping such confounding variables as size, speed, and direction of motion constant.

Studies linking stimulus magnitudes (e.g., size, luminosity, speed) with distortions in the perception of their durations generally make use of information-processing models as the theoretical basis for the interpretation of their findings (see Grondin, 2010, for a tutorial review). Among these, the Scalar Timing Theory (STT; Gibbon, Church, \& Meck 1984), the information processing variant of the Scalar Expectancy Theory (Gibbon, 1977; see Allman, Teki, Griffiths, \& Meck, 2014, for a review), allows researchers to make testable predictions based on modulations in its components as a result of manipulations made in external stimuli (i.e. the input component). For instance, an increase in pacemaker rate (i.e., due to arousal) is thought to underlie time dilation (Kaneko \& Murakami, 2009; Matthews, 2011; Zelkind, 1973; see Wearden, Edwards, Fakhri, \& Percival, 1998, for a discussion). Modulations of the attentional gating of pacemaker outputs to the working memory system or memory processes also have clear predictions regarding timing behavior (Droit-Volet, Clément, \& Wearden, 2001; Wearden et al., 1998). More specifically, it has been suggested that more attention paid to time should lead to more pulses being integrated in the clock stage (Zakay \& Block, 1995), which in turn code for a longer duration (i.e., dilation of perceived time; Yarrow,
Haggard, \& Rothwell, 2004). Thus, models such as the STT have consistently proven useful in explaining variations in timing behavior as a result of experimental manipulations.

Although current models of interval timing do not make clear predictions regarding SNR (motion coherence in our case) and perceived time, certain hypotheses can be formulated with regard to the cited literature on the effect of motion direction SNR on different components of temporal information processing. Principally, if the amount of coherent motion (i.e., SNR) in an RDM stimulus is taken as an indicator of the magnitude of the motion, an RDM stimulus with higher coherence would be expected to increase the rate of the pacemaker, leading to overestimations of durations coded by these stimuli, and vice versa. If, on the other hand, higher SNR grabs more attention at the expense of attention paid to the duration of the stimulus (Thomas \& Cantor, 1978; Thomas \& Weaver, 1975; Tse, Intriligator, Rivest, \& Cavanagh, 2004), then an RDM stimulus with higher coherence should decrease the rate of temporal integration, leading to underestimations of durations coded by these stimuli. Both of these effects would be multiplicative and therefore would be expected to be proportional to the target duration. If the presence of motion in the RDM stimulus introduces an additive effect (e.g., due to the delay in switch closure), however, its time normalized effect would decrease with longer target durations. In any case, the slope and intercept of regression lines relating reproduced durations to target durations can be used for capturing the effect of various types of experimental manipulations on disparate STT components (e.g., Wearden et al., 1998).

Finally, in addition to being a viable tool for representing various levels of SNR, the nature of the tobe-timed stimulus used in this study (i.e., the RDM stimulus) is also unique in the sense that the perception of different levels of SNR might correlate with the emergence of subtly different visual responses. More specifically, it can be assumed that various levels of embedded coherent motion might elicit different patterns of eye movements (Beutter \& Stone, 2000; Schütz, Braun, Movshon, \& Gegenfurtner, 2010; see Schütz, Braun, \& Gegenfurtner, 2011, for a review). These patterns in turn can be quantified by the number of exploratory saccades in response to (i.e., while observing) an RDM stimulus. This assumption has crucial implications for the current study because, in addition to being classically related to distortions in the perception of space (Lappe, Awater, \& Krekelberg, 2000; Ross, Morrone, \& Burr 1997), saccadic eye movements have recently been shown to compress perceived durations (see Burr et al., 2010; Eagleman, 2005). This, in turn, suggests that different patterns/ numbers of eye movements in response to different levels 
of motion coherence may also mediate systematic distortions of perceived durations (e.g., Cheng \& Penney, 2015; Penney et al., 2016).

Studies investigating the relationship between eye movements and time perception have tended to use highly controlled single trial paradigms, where the perceived duration between two eye movements was shown to be compressed as a result of single voluntary saccades (Morrone, Ross, \& Burr, 2005; Suzuki \& Yamazaki, 2010). In contrast, by employing an RDM stimulus as the to-be-timed stimulus, the current paradigm allows the participants to make multiple voluntary/exploratory saccades throughout a trial, thereby making it possible to observe the cumulative effect of a series of saccadic eye movements on perceived durations. In light of the cited literature, it was predicted that a duration timed concurrent with a higher number of saccades should be perceived to be shorter, compared to a duration in which a lower number of saccades were elicited by the stimulus. Thus, if the higher motion coherence elicits a larger number of eye movements, then it can be hypothesized, that within the temporal reproduction paradigm (Eisler, 1976), timed stimuli should be overreproduced if that duration has been encoded with lower coherence stimulus and reproduced with higher coherence stimulus. An inverse behavioral output (i.e., under reproduction of durations) is predicted when higher coherence stimulus during encoding is followed by a lower coherence stimulus during reproduction.

\section{Experiment 1}

\section{Method}

Participants Forty-one students of Koç University (18 male, $M_{\text {age }}=20.7$ years, age range $=18-26$ years) participated in Experiment 1 for course credit. Thirty-nine participants were tested in two identical sessions, whereas the remaining two participants were tested in a single session. Approximately $11 \%$ of all participants were left-handed. All experiments were approved by the Institutional Review Panel for Human Subjects of Koç University and were in accordance with the principles of the Declaration of Helsinki. All participants provided written consent for their participation for all three experiments.

Stimuli and apparatus Stimuli used were circular RDM patches, with a diameter of approximately $7.6 \mathrm{~cm}$, consisting of a percentage of randomly moving white dots $(3 \times 3$ pixels $)$ complemented by a coherent motion of the remaining dots (i.e., signal) in rightward or leftward direction (0 or 180 degrees, respectively). All stimuli were presented on a black background, on a 21 -in. LCD screen (60 Hz refresh rate) on an Apple iMac G4 computer, generated in MATLAB, using the PsychToolbox Extension (Brainard, 1997; Kleiner, Brainard, \& Pelli, 2007; Pelli, 1997) on the SnowDots framework developed by Joshua Gold at the University of Pennsylvania. Participants sat at a distance of 58-63 cm from the screen, in a dimly lit room, and provided their responses using a standard Apple iMac keyboard. In addition to three different coherences in the RDM stimuli $(0,8, \& 64 \%$; see below), an additional static stimulus was also used, which consisted of a snapshot image of a sample RDM patch, where none of the dots moved. No feedback was given in any of the experimental trials.

Procedure Participants' task was to reproduce a given (i.e., encoded) duration by holding down the space button as close to the target duration as possible. At the start of the first session, nine practice trials were completed, in which visual feedback was given as the normalized distance between a central red line representing the encoded duration, and a white line to the right or left of this central line, representing the amount of over- or underreproduction, respectively. Each trial began with the press of the space key, triggering the presentation of an RDM stimulus with 0,8 , or $64 \%$ coherent motion, or the static image, for a duration of $2.1,3.7$, or 5.4 seconds. Our main objectives in selecting these target durations were to (1) span a large enough supra-second duration range and (2) not present durations that can be sectioned into full seconds to prevent the use of any chronometric methods. After an interstimulus interval sampled from a truncated exponential distribution with a mean of $2 \mathrm{~s}$ (with a lower bound of $1 \mathrm{~s}$ and an upper bound of $5.6 \mathrm{~s}$ ), a short verbal instruction appeared on the screen, prompting participants to hold down the space key for reproducing the encoded duration. At the onset of this button press, another RDM stimulus was presented on the screen with one of the coherence levels until the space key was released. The presentation of the encoded RDM stimulus and its subsequent reproduction constituted a single trial. All coherence pairs - encoded and reproduced - and all durations were counterbalanced. Each session lasted 50-60 minutes. To further make sure that participants were looking at the screen and thereby paying attention to the task, in approximately $20 \%$ of the trials a small triangle or a square with a height of 10 pixels was flashed for $10 \mathrm{~ms}$ in the center of the screen, following the encoded stimulus. The participants' additional task in these trials was to report which shape was the one that was flashed. These trials were automatically replaced by others with identical settings (without flashing shape) at a random order within the session. Data from these replacement trials were used in the analyses instead. Each session consisted of a total of 230 trials, 192 of which were used in the analysis.

Data analysis Trials in which the reproduced durations were larger than 3 times, or less than one third of the target duration, were treated as outliers and excluded from the analysis 
(average percentage of cases, short duration: $M=2.87 \%$; mid duration: $M=2.52 \%$; long duration: $M=2.35 \%$ ). Participants with mean reproductions above and below two standard deviations of the sample mean in any of the target duration conditions were excluded from further analyses (amounting to four participants in this experiment).

A two-way repeated-measures ANOVA, with same coherence pairs (four levels: 0 vs. $0 ; 8$ vs. $8 ; 64$ vs. 64 ; static vs. static) and target duration (three levels: $2.1 \mathrm{~s}, 3.7 \mathrm{~s}, 5.4 \mathrm{~s}$ ) as withinsubjects factors, and mean normalized reproduction times as the dependent variable, was conducted. Additionally, a threefactor repeated-measures ANOVA was conducted, with duration (three levels: $2.1 \mathrm{~s}, 3.7 \mathrm{~s}, 5.4 \mathrm{~s}$ ), unequal coherence pairs (three levels: 0 vs. $8 ; 8$ vs. $64 ; 0$ vs. 64), and the order of lower coherence stimulus (two levels: lower coherence stimulus encoded \& lower coherence stimulus reproduced) as the within-subjects factors, and normalized reproduction time as the dependent variable, was conducted. If an interaction was observed between target duration and any of the withinsubjects factors, follow-up two-way repeated-measures ANOVAs, with coherence pairs (three levels) and the order of lower coherence stimulus (two levels) as the two factors, were conducted separately for the three target durations. Greenhouse-Geisser correction was applied for comparisons where sphericity was violated in all three experiments.

Finally, the change in normalized reproduction times of each participant were calculated and ordered as a function of the absolute difference between the coherence of the encoded and reproduced RDM stimulus, separately for the different orders of the lower coherence conditions and durations, and the gathered slopes were compared to a slope of 0 (i.e., slope expected if there was no effect of coherence difference) using one-sample $t$ tests.

By design, all three experiments in this study employed a variety of SNR levels. Namely, the coherence used in either the encoded or the reproduced stimulus order is of directly relevance to how it should be interpreted. We will use an order-specific parenthetical notation to refer to the coherence level of the encoded (i.e., first) and the reproduced (i.e., second) stimuli pairs within a trial. For instance, "( 8,64$)$ " will mean that "an RDM stimulus with $8 \%$ coherence was encoded, and an RDM stimulus with $64 \%$ coherence was reproduced."

\section{Results}

Although comparisons regarding differing encoded and reproduced coherence pairs were our main interest in this experiment, we first analyzed mean normalized reproduced durations (i.e., reproduced duration divided by target duration) in same coherence pairs across target durations to detect any systematic over- or underreproduction between durations (see Fig. 1). Visual inspection of Fig. 1a suggests an overreproduction of the 2.1-s duration, close-to-target reproduction of the 3.7-s duration, and an underreproduction of the 5.4-s duration (in all same-coherence pairs). Our analysis showed a main effect of target duration, $F(1.089,39.202)=$ $108.57, p<.001, \eta_{\mathrm{p}}^{2}=.75$, as well as a significant main effect of same coherence pairs, $F(3,108)=35.65, p<.001, \eta_{\mathrm{p}}^{2}=.50$, and no interaction between target duration and same coherence pairs, $F(4.05,145.75)=2.36, p=.055$. Post hoc analyses showed that the difference between all three durations reached significance (all $p \mathrm{~s}<.001$ ). Additionally, post hoc analyses of the effect of coherence pairs showed that the static dot array stimuli were reproduced significantly longer $(M=1.08)$ compared to all of the remaining stimuli with embedded coherent motion (i.e., 0, 8, \& $64 \%$ coherence stimuli; see Fig. 1a). Further analyses with unequal coherence pairs showed that, regardless of the order in which it was presented (i.e., during encoding; $M=1.07, S D=0.15$, or reproduction $M=1.06, S D$ $=0.15)$, the trials in which the static stimulus was presented were always overreproduced compared to trials in which both stimuli had embedded motion in them $(M=1, S D=0.14)$. Hence, although the static dot array stimulus was included in our study as a representative condition for an "absolute lack of motion," altogether these results preclude us from making further comparisons using the static dot array stimulus as a parametric level of SNR in combination with the three dynamic RDM stimuli. Therefore the remaining analyses were conducted by using those trials where both given and reproduced durations were represented by dynamic RDM stimuli only.

Next, we compared the data from unequal coherence pairs. The three-way repeated-measures ANOVA, with data from unequal coherence pair conditions, showed that all three main effects were significant, duration: $F(1.08,38.69)=107.05, p<$ $.001, \eta_{\mathrm{p}}^{2}=.75$; coherence pairs: $F(2,72)=20.31, p<.001, \eta_{\mathrm{p}}^{2}$ $=.36$; order of lower coherence stimulus: $F(1,36)=17.29, p<$ $.001, \eta_{\mathrm{p}}^{2}=.32$. There was also a significant interaction between duration and order of lower coherence, $F(1.43,51.52)$ $=4.55, p=.025, \eta_{\mathrm{p}}^{2}=.11$, as well as order of lower coherence and coherence pairs, $F(1.6,57.8)=8.77, p=.001, \eta_{\mathrm{p}}^{2}=.20$. For easier tractability, these results necessitated separate twoway ANOVAs to be conducted for the three target durations (see Table 1).

Note that if the SNR of the stimuli (or the difference between them) had no effect on the reproduced durations, we would expect no significant differences between the unequal encoded and reproduced coherence pairs when the order of stimuli is switched. Our analyses opposed this prediction,

Fig. 1 Mean normalized reproduction time as a function of same coherence pairs and target duration in (a) Experiment 1, (b) Experiment 2, and (c) Experiment 3. Dashed green horizontal line denotes hypothetical perfectly accurate performance. Error bars denote standard errors of the mean. (Color figure online) 


\section{a}

Experiment 1

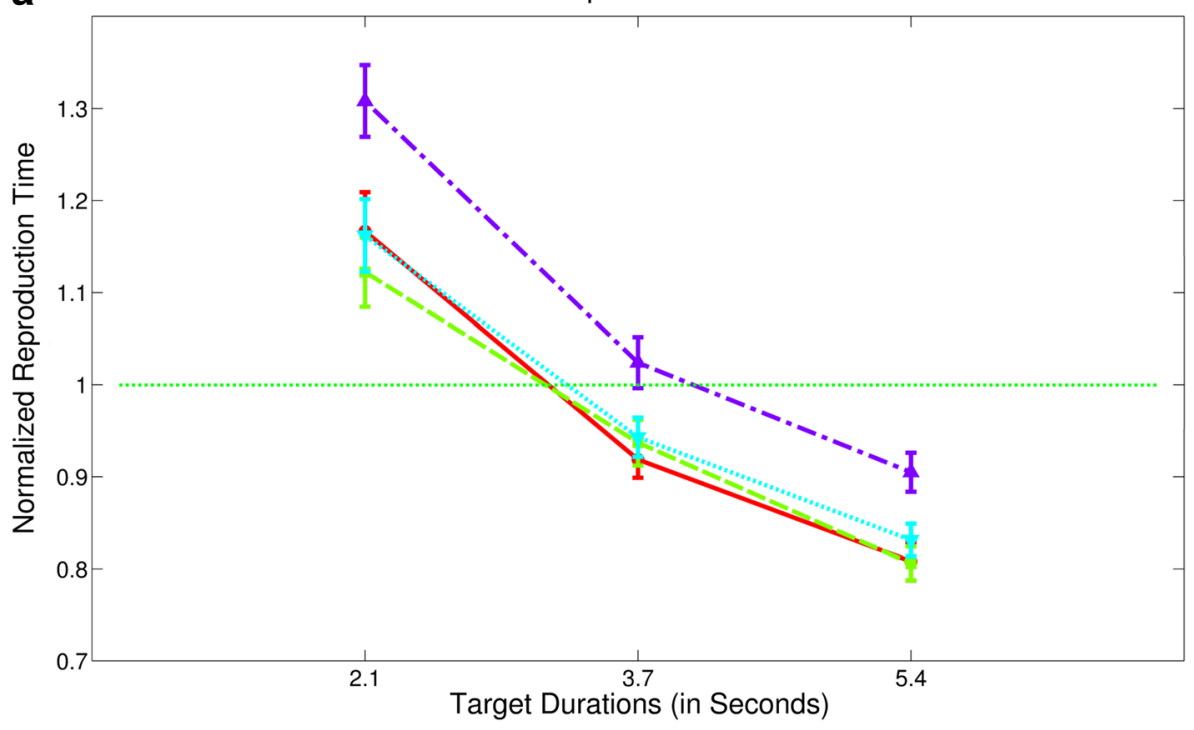

$-(8,8)$

$-(23,23)$

- - $(64,64)$

$\cdot-\cdot(98,98)$

b

Experiment 2

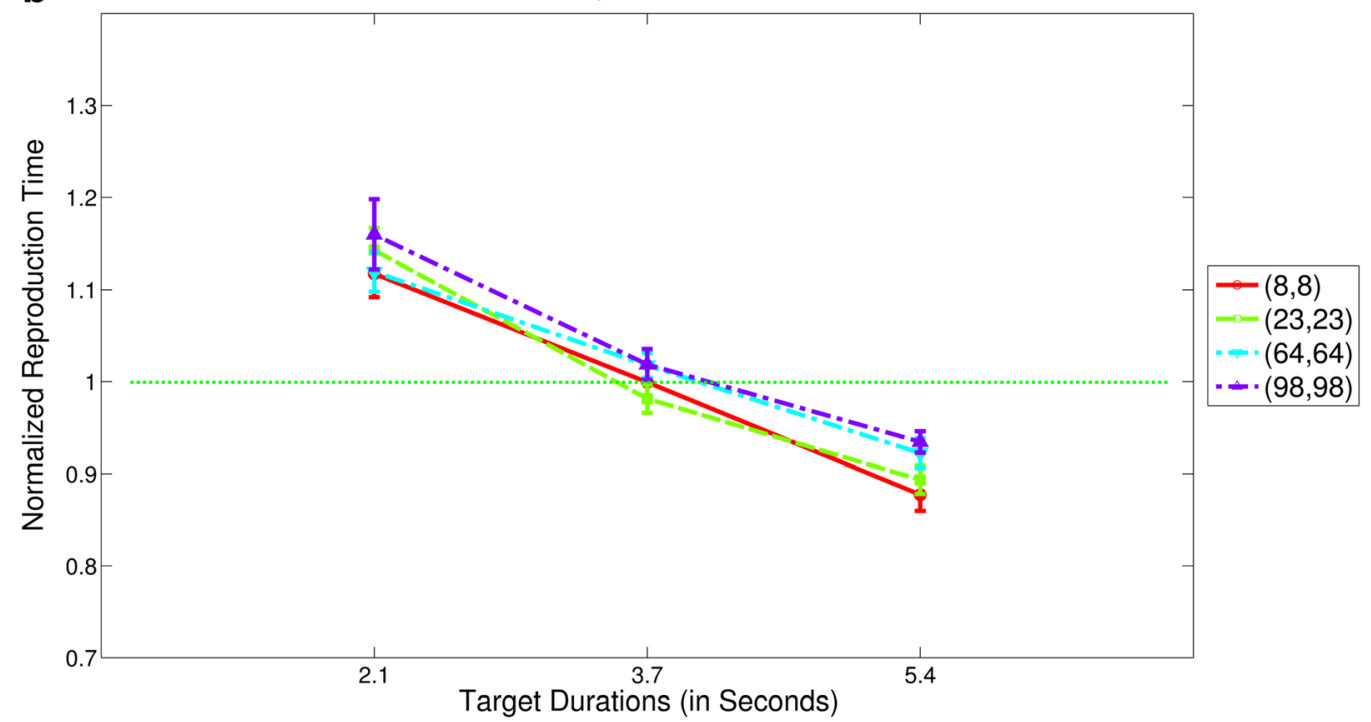

C

Experiment 3

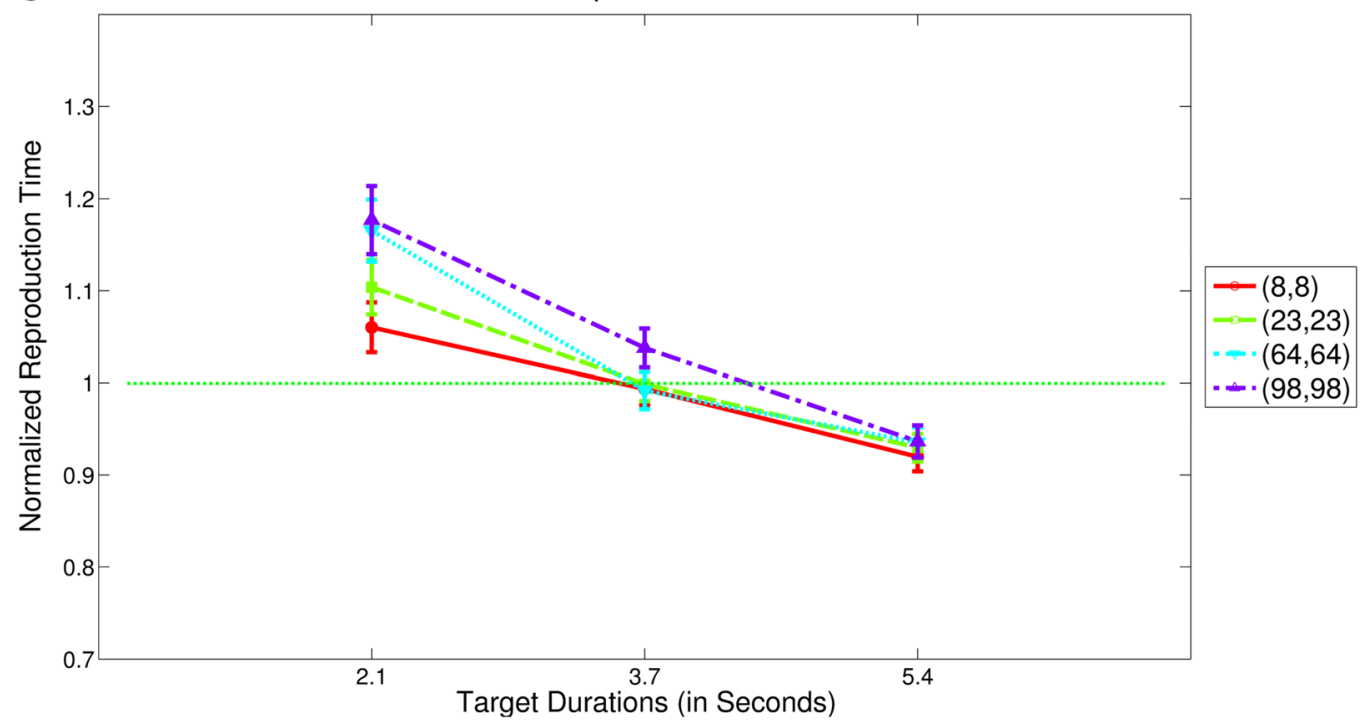


Table 1 Order of lower coherence stimulus $\times$ unequal coherence pairs repeated-measures ANOVA for normalized reproduced duration, for three target duration conditions (2.1, 3.7, \& 5.4 seconds), in Experiment 1

\begin{tabular}{llllll}
\hline Target duration & Source & $d f$ & $F$ & $\eta_{\mathrm{p}}^{2}$ & $p$ \\
\hline 2.1 seconds & (A) Order of lower coherence & 1 & 11.56 & .24 & .002 \\
& (B) Unequal coherence pairs & 2 & 10.24 & .22 & .001 \\
& A $\times$ B (interaction) & 2 & 2.35 & .06 & .1 \\
& Error (within subjects) & 72 & & & \\
3.7 seconds & (A) Order of lower coherence & 1 & 13.21 & .27 & .001 \\
& (B) Unequal coherence pairs & 1.63 & 8.53 & .19 & $.001^{\text {a }}$ \\
& A $\times$ B (interaction) & 2 & 7.71 & .18 & .001 \\
& Error (within subjects) & 72 & & & \\
5.4 seconds & (A) Order of lower coherence & 1 & 5.15 & .13 & .03 \\
& (B) Unequal coherence pairs & 2 & 7.9 & .18 & .001 \\
& A $\times$ B (interaction) & 2 & 4.16 & .1 & .02 \\
& Error (within subjects) & 72 & & & \\
\hline
\end{tabular}

${ }^{a}$ Greenhouse-Geisser correction.

showing that the reproduced durations were significantly longer when the lower coherence is encoded compared to when it is reproduced for all target durations (see Table 1). There was also a significant effect of unequal coherence pairs for all durations, as well as an interaction between the coherence pairs and low coherence order for the 3.7 and $5.4 \mathrm{~s}$ duration conditions (see Table 1). The simple effects analyses of this interaction effect showed that, for both target duration conditions, there was an effect of lower coherence order in the $(8$, $64)$ and $(0,64)$ pairs (all $p s<.05)$, and no significant difference in the $(0,8)$ pair was observed (both $p \mathrm{~s} \geq .27$ ), which suggests that mean reproduced durations were longer for "lower encoded" coherence pairs when the difference between the coherences was large for the longer durations (see Fig. 2b-c). Results depicted in Table 1 suggest an effect of coherence pairs for all reproduced durations.

To better characterize the relationship between the direct index of change in reproduced durations and the difference between encoded and reproduced coherence pairs, we conducted a regression analysis. To this end, the change in reproduction with increasing difference in coherence was quantified as the slope of the lines (i.e., the regression equation) that relate the reproduced durations to the lower coherence encoded and lower coherence reproduced trial conditions of these coherence pairs (see Fig. 2, solid red and dashed blue lines, respectively). Our comparisons of participants' slopes to a value of 0 for the low coherence (i.e., SNR) encoded/high coherence reproduced, and the high coherence encoded/low coherence reproduced conditions (see Fig. 2a-c) showed that in the lower coherence encoded condition, the slopes differed significantly from 0 for all test durations; $2.1 \mathrm{~s}(M=0.06, S D$ $=0.12), t(36)=2.93, p=.006,3.7 \mathrm{~s}(M=0.04, S D=0.06)$, $t(36)=3.68, p<.001$, and $5.4(M=0.02, S D=0.04), t(36)=$
$3.45, p=.001$, target durations, whereas the slopes of the lower coherence second condition in the $2.1 \mathrm{~s}(M=0.01, S D$ $=0.09), 3.7 \mathrm{~s}(M=0.003, S D=0.05)$, or the $5.4 \mathrm{~s}(M=-0.005$, $S D=0.05)$ target duration conditions were not significantly different from the slope of 0 (all $p \mathrm{~s} \geq .36$ ).

The same analyses were conducted for participants' coefficient of variation $(\mathrm{CV})$ values. CVs were calculated by dividing each participant's standard deviation of reproduction durations by their mean. Results showed that target duration had a significant effect on CVs, $F(1.56,55.99)=30.54, p<$ $.001, \eta_{\mathrm{p}}^{2}=.46$, where $\mathrm{CVs}$ in the $2.1 \mathrm{~s}$ condition $(M=.251)$ were significantly higher compared to the $3.7 \mathrm{~s}(M=.193)$ and $5.4 \mathrm{~s}(M=.196)$ conditions (both $p \mathrm{~s}<.001)$, whereas the difference between the latter two conditions did not reach significance $(p=.58)$. Coherence pairs or the order of lower coherence stimulus had no effect on CVs (both $p \mathrm{~s} \geq .44$ ). Identical results were obtained with analyses conducted using same coherence pairs.

To determine if the previously observed effect of SNR on reproduced durations is multiplicative (i.e., proportional to the target duration) or additive, or potentially a mixture of the two, we have calculated the mean absolute differences in raw (i.e., nonnormalized) reproduced durations between low coherence encoded and low coherence reproduced conditions for each participant, separately for each target duration (i.e., mean difference between solid red and dashed blue lines in Fig. 2a-c). The differences in reproduced durations were $M=0.258$ for the short duration, $M=0.259$ for the mid duration, and $M=$ 0.243 for the long duration. One-way repeated-measures ANOVA did not reveal a significant effect of target duration on the coherence order-based difference in reproduced durations $(p=.89)$. These results point at the additive nature of the observed effects. To further elucidate the question of proportionality and additivity of the effects, we have also calculated the slope and intercept of the regression lines relating these difference scores to target durations for each participant. Next, one-sample $t$ tests were conducted to compare these two parameter values to a value of 0 . A slope value significantly higher than 0 was hypothesized to capture the multiplicative portion of the effect of the SNR, whereas a nonzero intercept value would capture the additive portion of the effect of the SNR, which applies equally to all durations. Results suggest that the although the intercept of the regression line was significantly higher than $0(M=0.27, S D=0.338, t(36)=4.87, p$ $<.001)$, the slope $(M=-0.005)$ was not $(p=.14)$.

\section{Experiment 2}

We modified the experimental design in Experiment 2 to test the generality and robustness of the findings in Experiment 1 by testing a different group of participants with a different set of SNRs (i.e., motion coherences). 


\section{Experiment 1}
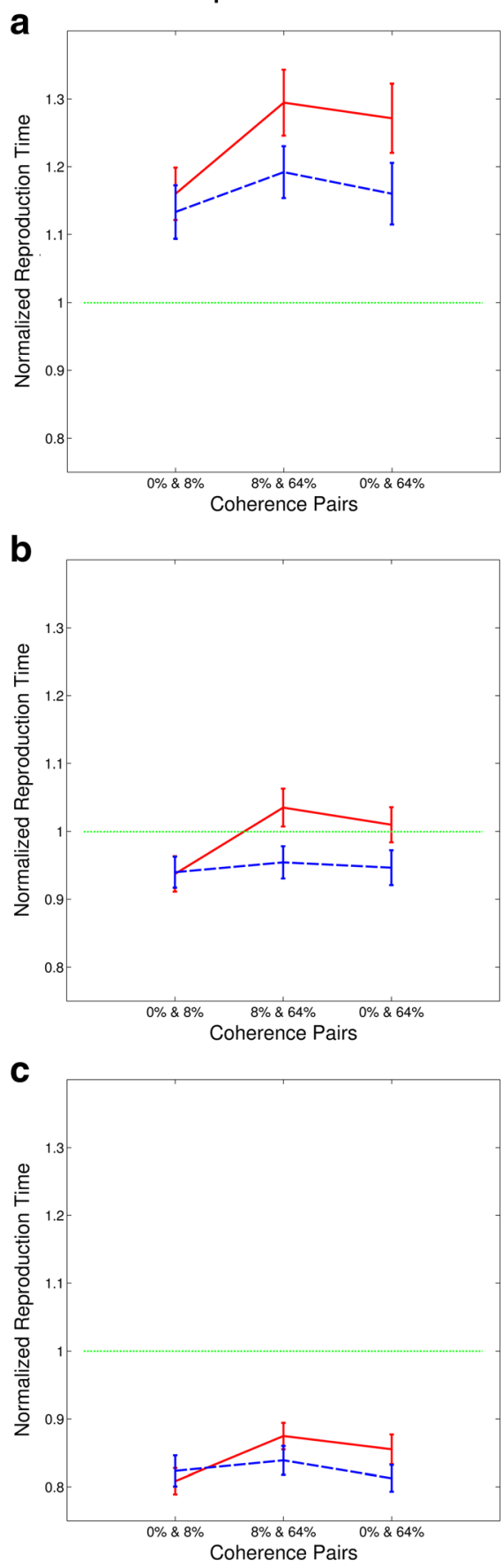

Fig. 2 Mean normalized reproduced durations as a function of coherence pair and the order of lower coherence in Experiment 1 (a-b-c) and Experiment 2 (d-e-f) in 2.1 second (a \& d), 3.7 second (b \& e), and 5.4 second $(\mathbf{c} \& \mathbf{f})$ conditions. Coherence pairs are ordered in ascending order

\section{Method}

Participants The inclusion criteria for participants in Experiment 2 were the same as those in Experiment 1. Thirty-six participants were tested in Experiment 2 (12 male, $M_{\text {age }}=19.8$ years, age range $=18-23$ years $), 29$ of which

\section{Experiment 2}
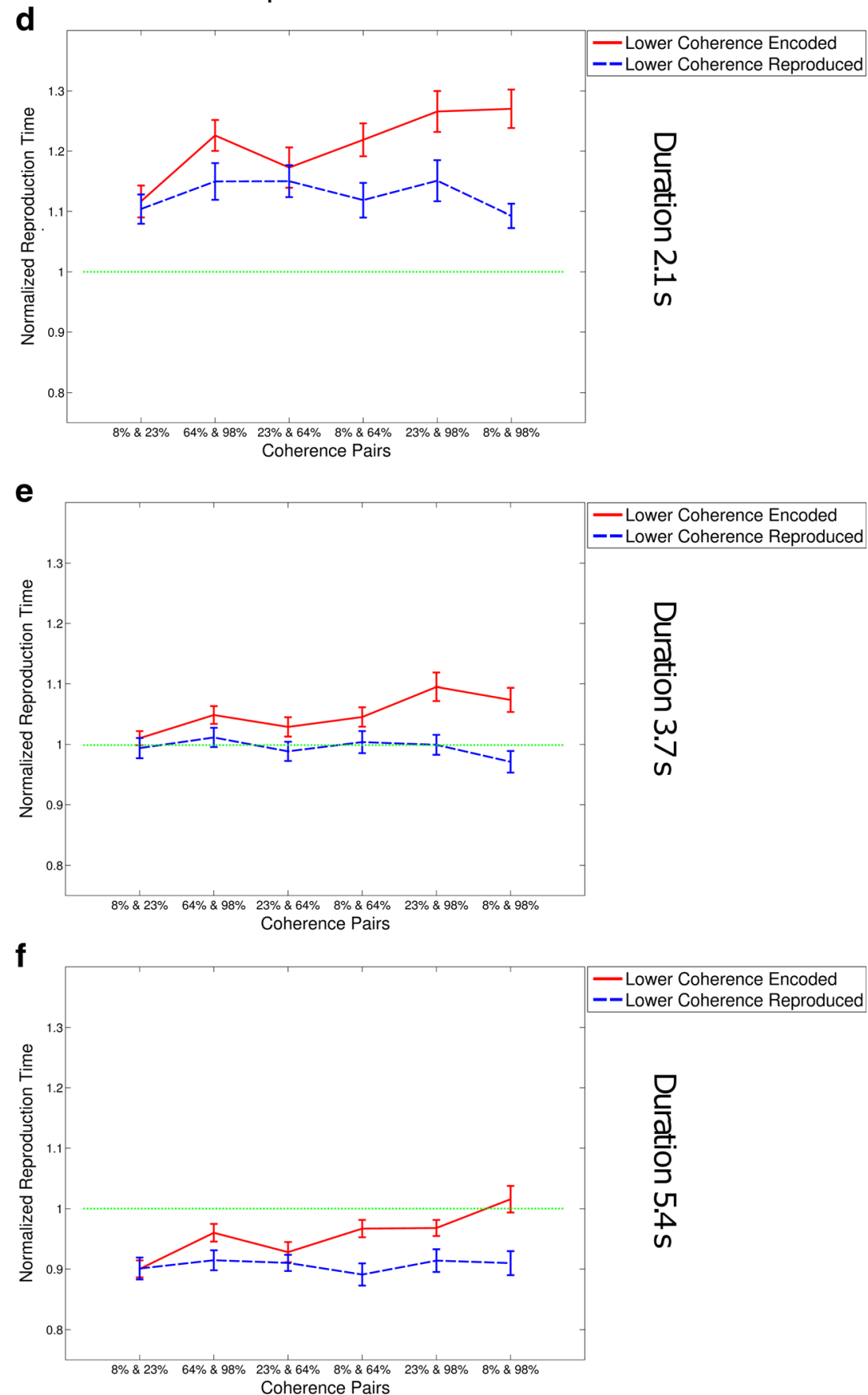

according to their difference. Dashed green horizontal line denotes hypothetical perfectly accurate performance. Error bars denote standard errors of the mean. (Color figure online)

participated for course credit in two identical sessions, whereas the remaining seven participated in a single session.

Procedure The procedure of Experiment 2 was identical to Experiment 1, except for the replacement of the $0 \%$ coherence stimulus and the static stimulus with 23 and $98 \%$ 
coherence stimuli. This change was implemented for three reasons: (1) The 0 and $8 \%$ coherence stimuli were generally reported to be perceptually very similar by the participants in Experiment 1, (2) the static stimulus was systematically overestimated and therefore could not be treated as a parametric level of SNR to analyses, and (3) we aimed to explore the effect of a wider range of coherence levels for completeness.

Data analysis As with Experiment 1, trials in which reproduced durations were larger than 3 times, or less than one third of the target duration were excluded as outliers (average percentage of cases: short duration: $M=2.1 \%$; did duration: $M=2.55 \%$; long duration: $M=2.45 \%$ ). Additionally, five participants' data were excluded from analyses in line with the exclusion criterion described earlier (see Experiment 1, Data Analysis section). The same analyses described for Experiment 1 were also applied to the data gathered from this experiment.

\section{Results}

Consistent with the results of Experiment 1, our analysis of the mean normalized reproduced durations (i.e., reproduced duration divided by target duration) in same coherence pairs across target durations in Experiment 2 revealed a main effect of target duration, $F(1.177,35.312)=60.3, p<.001, \eta_{\mathrm{p}}^{2}=.67$, as well as a significant main effect of same coherence pairs, $\left(F(2.1,62)=3.326, p=.041, \eta_{\mathrm{p}}^{2}=.10\right.$, and no interaction between target duration and same coherence pairs $(p=.24)$. Post hoc analyses based on the normalized reproduction times showed that the difference between all three durations reached significance (all $p \mathrm{~s}<.001$; see Fig. 1b). Additionally, post hoc analyses of the effect of coherence pairs showed that the $(8,8)$ coherence pair $(M=1)$ was reproduced significantly shorter than the $(98,98)$ coherence pair $(M=1.04, p=.012)$. None of the remaining comparisons reached significance (all $p s \geq .071$; see Fig. 1b).

The three-way repeated-measures ANOVA, conducted with unequal coherence pairs in Experiment 2, showed the identical pattern as that conducted in Experiment 1. Namely, all three main effects were significant, duration: $F(1.15$, $33.44)=78.13, p<.001, \eta_{\mathrm{p}}^{2}=.73$; coherence pairs: $F(5$, $145)=8.22, p<.001, \eta_{\mathrm{p}}^{2}=.22$; order of lower coherence stimulus: $F(1,29)=34.78, p<.001, \eta_{\mathrm{p}}^{2}=.55$. There was also a significant interaction between duration and order of lower coherence, $F(2,58)=7.07, p=.002, \eta_{\mathrm{p}}^{2}=.20$, as well as order of lower coherence and coherence pairs, $F(2.99,86.86)=$ $10.59, p<.001, \eta_{\mathrm{p}}^{2}=.27$.

Results of Experiment 2 regarding unequal coherence pairs for different duration conditions also closely resembled those obtained in Experiment 1 (see Table 2), where an increase in SNR from an encoded to a reproduced stimulus led to an
Table 2 Order of lower coherence stimulus $\times$ unequal coherence pairs repeated-measures ANOVA for normalized reproduced duration, for three target duration conditions (2.1, 3.7, \& 5.4 seconds), in Experiment 2

\begin{tabular}{llllll}
\hline Target duration & Source & $d f$ & $F$ & $\eta_{\mathrm{p}}^{2}$ & $p$ \\
\hline \multirow{2}{*}{2.1 seconds } & (A) Order of lower coherence & 1 & 42.36 & .59 & .001 \\
& (B) Unequal coherence pairs & 3.57 & 4.28 & .13 & $.004^{\mathrm{a}}$ \\
& A $\times$ B (interaction) & 5 & 7.43 & .2 & .001 \\
& Error (within subjects) & 145 & & & \\
3.7 seconds & (A) Order of lower coherence & 1 & 21.8 & .42 & .001 \\
& (B) Unequal coherence pairs & 5 & 3.4 & .1 & .006 \\
& A $\times$ B (interaction) & 2.77 & 3.23 & .1 & $.03^{\mathrm{a}}$ \\
& Error (within subjects) & 145 & & & \\
5.4 seconds & (A) Order of lower coherence & 1 & 16.89 & .36 & .001 \\
& (B) Unequal coherence pairs & 5 & 7.69 & .2 & .001 \\
& A $\times$ B (interaction) & 2.94 & 5.76 & .16 & $.001^{\mathrm{a}}$ \\
& Error (within subjects) & 145 & & & \\
\hline
\end{tabular}

${ }^{a}$ Greenhouse-Geisser correction.

overreproduction, and this effect was magnified with increasing difference between the encoded and the reproduced coherences. On the other hand, a decrease in SNR from a encoded to a reproduced stimulus did not result in an underreproduction of the target durations by the same amount (see Fig. 2d-f).

Table 2 shows that for all duration conditions there was a significant effect of coherence pairs, as well as a significant effect of the order of lower coherence stimulus, in addition to an interaction effect of these two factors. Simple effects analyses of the significant interaction in 2.1-s duration condition showed an effect of lower coherence order in the $(64,98),(8$, $64),(23,98)$, and $(8,98)$ pairs (all $p s<.001)$, in all of which the normalized reproduced durations were longer when the participants encoded the duration with the lower coherence (note that one participant's data in the $[23,8]$ pair were not included in the analysis due to exclusion criteria). Simple effect analyses in the 3.7-s duration condition showed an effect of lower coherence order in the $(64,98),(23,64),(23,98)$, and $(8,98)$ pairs (all $p \mathrm{~s}<.05)$, where the normalized reproduced durations were longer when lower coherence was encoded compared to when it was reproduced. Finally, in the post hoc comparisons of the interaction effect in the 5.4-s condition, the effect of order of lower coherence reached significance in the $(64,98),(8,64),(23,98)$, and $(8,98)$ pairs (all $p \mathrm{~s}$ $<.05)$, where the normalized reproduced durations were longer when the lower coherence was encoded compared to when it was reproduced.

Our comparisons of participants' slopes of the lines that relate the reproduced durations to the low SNR encoded/ high SNR reproduced, and the high SNR encoded/low SNR reproduced conditions (see Fig. 2d-f) showed the exact pattern as the one seen in Experiment 1. Namely, in the lower 
coherence encoded condition, the slopes differed significantly from a value of 0 in the 2.1-s $(M=0.03, S D=0.03), t(30)=$ $5.57, p<.001 ; 3.7-\mathrm{s}(M=0.01, S D=0.02), t(30)=4.26, p<$ .001 ; and 5.4-s $(M=0.02, S D=0.02), t(30)=5.08, p<.001$, target durations, whereas the slopes of the lower coherence second condition in the 2.1-s $(M=-0.004, S D=0.02), 3.7-\mathrm{s}$ $(M=-0.004, S D=0.02)$, or the 5.4-s $(M=0, S D=0.01)$ target durations were not significantly different from the slope of 0 (all $p \mathrm{~s} \geq .35$ ). (Note that the data of the same participant were excluded from the analysis.)

As with Experiment 1, a three-way ANOVA was performed to determine if mean $\mathrm{CV}$ values changed as a function of target duration, coherence pairs, or the order of lower coherence stimulus. Results showed that target duration had a significant effect on CVs, $F(2,60)=39.57$, $p<.001, \eta_{\mathrm{p}}^{2}=.57$. Post hoc pairwise comparisons showed that all duration pairs differed significantly from each other (all $p$ s $<.01$ ), with CVs being highest in the 2.1-s condition $(M=.19)$, followed by the 3.7-s $(M=.154)$ and the 5.4-s $(M=.137)$ conditions. Additionally, there was a main effect of the order of lower coherence stimulus on $\mathrm{CV}, F(1,30)=4.58, p=.04, \eta_{\mathrm{p}}^{2}=.13$, where $\mathrm{CVs}$ were higher when lower coherence was reproduced $(M=$ $.165)$, compared to when it was encoded $(M=.155)$. There was no main effect of coherence pairs, or a significant interaction between any of the factors on CV (all $p$ s $\geq$.13). (Results obtained with analyses using same coherence pairs were the same, except that they showed that CVs in the 3.7-s and 5.4-s conditions did not differ significantly.)

Also identical with Experiment 1, the mean absolute differences in raw reproductions between low encoded and low reproduced conditions (i.e., mean difference between solid red and dashed blue lines in Fig. 2d, e, \& f) were calculated for each participant, separately for each target duration. A one-way repeated-measures ANOVA, with this difference value as the dependent and target duration as the independent variable, was conducted to determine if the previously observed effect of SNR on reproduced durations is multiplicative or additive (or potentially a mixture of the two). Results revealed a significant effect of target duration on the coherence orderbased difference in reproduced durations, $F(2,60)=$ $6.92, p=.002, \eta_{\mathrm{p}}^{2}=.19$. Post hoc analyses revealed that the effect of SNR on reproduced durations tended to increase with longer target durations $\left(M_{\text {short }}=0.191, M_{\text {mid }}=\right.$ $0.23, M_{\text {long }}=0.332$ ). All comparisons, except for the one between the mid and long target duration conditions ( $p=$ .21 ), reached significance (all $p$ s $<0.05$ ). Additionally, we calculated the slope and intercept of the regression lines relating these absolute difference scores to target durations for each participant. One-sample $t$ tests suggest that both the slope $(M=0.043, S D=0.075), t(30)=3.18$, $p=.003$, and the intercept of the regression line were significantly higher than $0(M=0.09, S D=0.221)$, $t(30)=2.28, p=.03$.

\section{Experiment 3}

Experiments 1 and 2 provided almost identical results regarding the behavioral effect of coherent motion of perceived time, where overreproduction of durations is observed when durations are encoded with lower and reproduced with higher coherence stimuli. In Experiment 3, we specifically aimed to investigate the potential relationship between this robust behavioral effect and the change in the number of eye movements from encoding to reproduction. Accordingly, Experiment 3 was, in effect, identical to Experiment 2, except for the addition of the eye-tracking methodology. A different group of participants were tested in this study.

\section{Method}

Stimuli and apparatus The experimental procedure and the stimulus properties used in Experiment 3 were identical to those used in Experiment 2. Participants' eye movements were recorded by a Tobii T120 eye tracker (Tobii Technology, AB, Danderyd, Sweden) at a constant frame rate of $120 \mathrm{~Hz}$ (at approximately 8-ms intervals) using five infrared lights. Stimuli were presented on the integrated 17 -inch TFT screen of the T120 $(1,280 \times 1,024$ pixel resolution). No head mount was used. Two nine-point calibrations were made: one in the beginning and another one halfway through the session.

Participants Thirty-seven participants participated in a single session (16 male, $M_{\mathrm{age}}=19.4$ years, age range $=18-24$ years), and were paid 15 liras for participation (approx. \$5).

Data analysis As with Experiment 1 and 2, trials in which the reproduced durations were larger than three times or less than one third of the target duration were excluded as outliers (average percentage of cases: short duration: $M=2.21 \%$; mid duration: $M=2.45 \%$; long duration: $M=2.3 \%$ ). Additionally, five participants' data were excluded from analyses in line with the exclusion criterion described previously (see Experiment 1, Data Analysis section). The behavioral analyses conducted in Experiment 3 were identical with those in Experiment 2 (see Experiment 2, Data Analysis section).

Preprocessing of eye tracking data was done by replacing each bad value with the last good value before it, as described in Leppänen, Forssman, Kaatiala, Yrttiaho, and Wass (2014). Bad values were determined based on the most conservative (exclusive) measure of gaze data validity as suggested by 
Tobii, where the eye-tracking system successfully recorded both eyes and was sure as to which eye the detected gaze data came from (invalid and uncertain frames during encoding: $M$ $=25.01 \%, S D=19.28 \%$; invalid and uncertain frames during reproduction: $M=26.58 \%, S D=20.28 \%$; see Nevalainen \& Sajaniemi, 2004, for reference values). Interpolated gaze data were then smoothed by applying a 5-point running average, after which the number of saccadic eye movements in each trial was calculated by the Microsaccade Toolbox for R (Engbert, Sinn, Mergenthaler, \& Trukenbrod 2015). Number of saccades in each trial, for both the encoded and the reproduced RDM stimuli, were then estimated by using the same toolbox. The velocity threshold for a saccade was set at 3 times the median of smoothed gaze data (see Engbert et al., 2015). Candidate saccade sequences had to pass this threshold for a minimum duration of two data samples (i.e., around 16.7 $\mathrm{ms}$ ), which were then identified as binocular saccades from monocular candidate sequences in right and left eyes (Engbert et al., 2015).

\section{Results}

Our analysis of the mean normalized reproduced durations in same coherence pairs across target durations in Experiment 3 revealed a main effect of target duration, $F(1.213,37,603)=$ $59.6, p<.001, \eta_{\mathrm{p}}^{2}=.67$, as well as a significant main effect of same coherence pairs, $F(2.104,65.234)=5.43, p=.006, \eta_{\mathrm{p}}^{2}=$ .15 , and a significant interaction between target duration and same coherence pairs, $F(3.92,121.36)=2.635, p=.038, \eta_{\mathrm{p}}^{2}=$ .08 . Post hoc analyses based on the normalized reproduction times showed that the difference between all three durations reached significance for all coherence pairs (all $p \mathrm{~s}<.01$; see Fig. 1c). Additionally, post hoc analyses of the effect of coherence pairs showed that the $(8,8)$ coherence pair $(M=1.06)$ was reproduced significantly shorter than the $(64,64)$ and the $(98,98)$ coherence pairs ( $M=1.165, M=1.177$, respectively), in the 2.1-s duration (both $p \mathrm{~s}<.01$ ), and the $(64,64)$ coherence pair $(M=0.992)$ was reproduced significantly shorter than the $(98,98)$ coherence pair $(M=1.038, p=.032)$ in the 3.4-s duration conditions. None of the remaining comparisons reached significance (all $p \mathrm{~s} \geq .061$; see Fig. 1c).

The three-way repeated-measures ANOVA conducted with unequal coherence pairs in Experiment 3 showed the identical pattern as that conducted in Experiments 1 and 2. Namely, all three main effects were significant, duration: $F(1.18,36.52)=$ $87.59, p<.001, \eta_{\mathrm{p}}^{2}=.74$; coherence pairs: $F(3.13,96.88)=$ $8.45, p<.001, \eta_{\mathrm{p}}^{2}=.21$; order of lower coherence stimulus: $F(1,31)=32.48, p<.001, \eta_{\mathrm{p}}^{2}=.51$. There was also a significant interaction between duration and order of lower coherence, $F(1.35,41.73)=21.37, p<.001, \eta_{\mathrm{p}}^{2}=.41$, as well as order of lower coherence and coherence pairs, $F(2.94,91.04)$ $=13.15, p<.001, \eta_{\mathrm{p}}^{2}=.30$.
Results of Experiment 3 regarding unequal coherence pairs for different duration conditions also closely resembled those obtained in Experiment 1 and 2, where an increase in SNR from an encoded to a reproduced stimulus lead to an overreproduction, and this effect was magnified with increasing difference between the encoded and the reproduced coherences (see Fig. 3). The statistical outputs are presented in Table 3.

Table 3 shows that, as with Experiment 2, for all duration conditions there was a significant effect of coherence pairs, as well as a significant effect of the order of lower coherence stimulus, in addition to an interaction effect of these two factors. Simple effects analyses of the significant interaction in both 2.1-s and 3.7-s conditions showed an effect of lower coherence order in the all coherence pairs, except for $(8,23)$ (all $p \mathrm{~s}<.05$ ), in all of which the normalized reproduced durations were longer when the participants encoded the duration with the lower coherence. Finally, simple effects analyses of the significant interaction in the 5.4-s condition showed an effect of lower coherence order in the all coherence pairs except for the $(8,23)$ and $(64,98)$ (all $p s<.05)$, in all of which the normalized reproduced durations were longer when the participants encoded the duration with the lower coherence.

Our comparisons of participants' slopes of the lines that relate the reproduced durations to the low SNR encoded/ high SNR reproduced, and the high SNR encoded/low SNR reproduced conditions (see Fig. 3a-c) showed the a similar pattern to the ones seen in Experiments 1 and 2. Namely, in the lower coherence encoded condition, the slopes differed significantly from a value of 0 in the 2.1-s $(M=0.04, S D=$ $0.04), t(31)=4.88, p<.001,3.7$-s $(M=0.02, S D=0.04), t(31)$ $=3.81, p=.001$, and 5.4-s $(M=0.02, S D=0.03), t(31)=4.15$, $p<.001$, target duration conditions, whereas the slopes of the lower coherence second condition in the 3.7-s $(M=-0.003$, $S D=0.02)$ or the $5.4-\mathrm{s}(M=0.001, S D=0.02)$ target duration conditions were not significantly different from the slope of 0 (both $p \mathrm{~s} \geq$. 46). The slope of the lower coherence reproduced condition in the 2.1-s condition did, however, significantly differ from $0(M=-0.013, S D=0.03), t(31)=-2.5, p<.001$.

As with Experiments 1 and 2, a three-way ANOVA was conducted to see if CV values differed significantly, depending on reproduced duration, unequal coherence pairs, or the order of lower coherence stimulus. Results suggest a significant effect of target duration on CV, $F(2,62)=44.17, p<.001$, $\eta_{\mathrm{p}}^{2}=.59$. Post hoc analyses parallel those in Experiment 2, with differences in all target duration pairs reaching significance (all $p \mathrm{~s}<.001$ ). Specifically, CVs decreased with

Fig. 3 Mean normalized reproduced durations as a function of coherence pair and the order of lower coherence in Experiment 3. Coherence pairs are ordered in ascending order according to their difference. Dashed green horizontal line denotes hypothetical perfectly accurate performance. Error bars denote standard errors of the mean. (Color figure online) 
a

Target Duration $2.1 \mathrm{~s}$

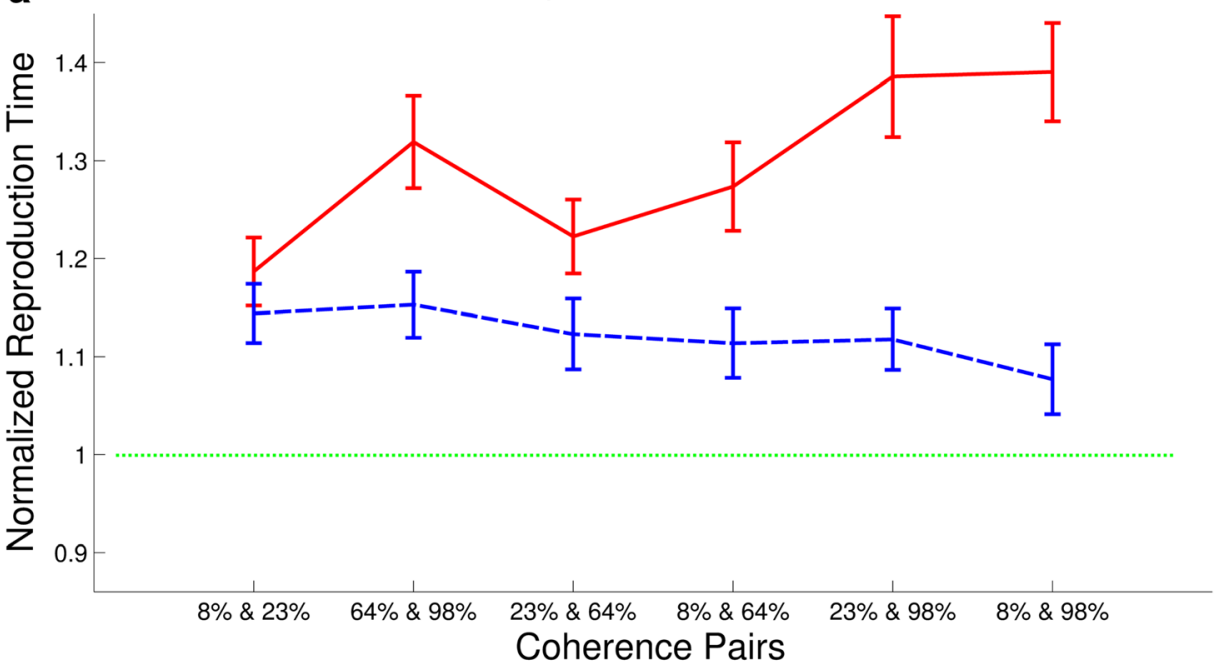

b

Target Duration $3.7 \mathrm{~s}$

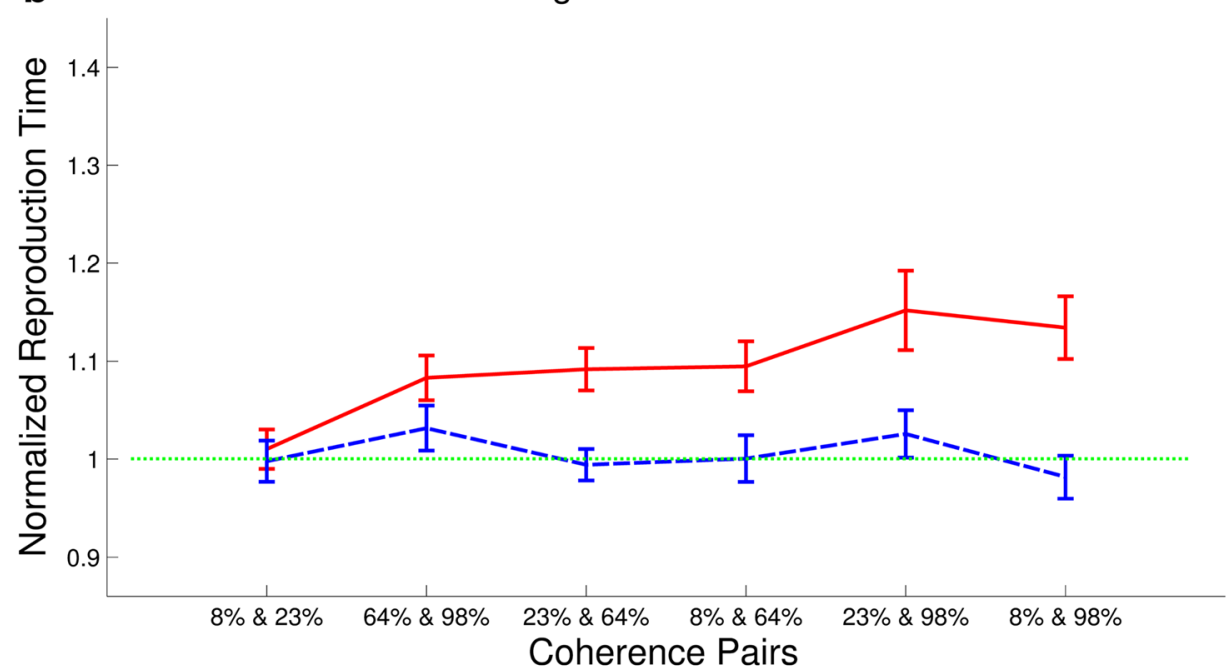

C

Target Duration $5.4 \mathrm{~s}$

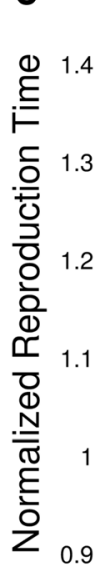

4

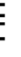

든 1.3

$1.2-$

1.1
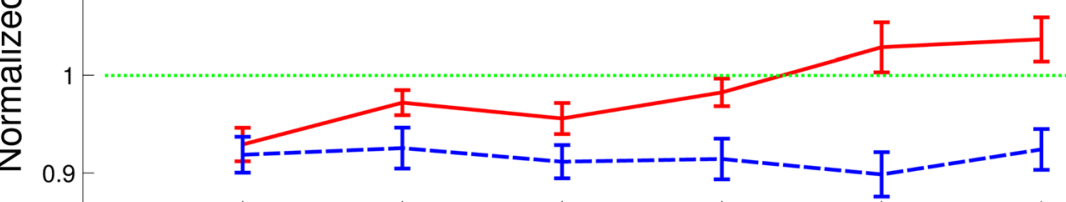

$8 \% \& 23 \% \quad 64 \% \& 98 \% \quad 23 \% \& 64 \% \quad 8 \% \& 64 \% \quad 23 \% \& 98 \% \quad 8 \% \& 98 \%$

Coherence Pairs

- Lower Coherence Encoded

--Lower Coherence Reproduced 
Table 3 Order of lower coherence stimulus $\times$ unequal coherence pairs repeated-measures ANOVA for normalized reproduced duration, for three target duration conditions (2.1, 3.7, \& 5.4 seconds), in Experiment 3

\begin{tabular}{llllll}
\hline Target duration & Source & $d f$ & $F$ & $\eta_{\mathrm{p}}^{2}$ & $p$ \\
\hline \multirow{2}{*}{ 2.1 seconds } & (A) Order of lower coherence & 1 & 33.89 & .52 & .001 \\
& (B) Unequal coherence pairs & 5 & 4.05 & .12 & .002 \\
& A $\times$ B (interaction) & 3.78 & 8.18 & .21 & $.001^{\text {a }}$ \\
& Error (within subjects) & 155 & & & \\
3.7 seconds & (A) Order of lower coherence & 1 & 27.34 & .46 & .001 \\
& (B) Unequal coherence pairs & 5 & 5.22 & .14 & .001 \\
& A $\times$ B (interaction) & 3.72 & 3.52 & .1 & $.011^{\text {a }}$ \\
& Error (within subjects) & 155 & & & \\
5.4 seconds & (A) Order of lower coherence & 1 & 19.57 & .39 & .001 \\
& (B) Unequal coherence pairs & 3.38 & 3.55 & .1 & .014 \\
& A $\times$ B (interaction) & 3.47 & 5.02 & .14 & .002 \\
& Error (within subjects) & 155 & & & \\
\hline
\end{tabular}

${ }^{\text {a }}$ Greenhouse-Geisser correction.

increasing target duration, with highest $\mathrm{CV}$ s observed in the 2.1-s duration condition $(M=.19)$, followed by the 3.7-s $(M=$ $.138)$ and the 5.4 -s duration conditions $(M=.126)$, respectively. None of the other main or interaction effects reached significance (both $p s \geq$. 64). Identical results were obtained with analyses conducted using same coherence pairs.

Finally, as with Experiments 1 and 2, to determine if the previously observed effect of SNR on reproduced durations is multiplicative or additive, a one-way repeated-measures ANOVA was conducted, with mean absolute raw differences in reproduced durations for the three target duration conditions as the dependent variable, and target duration as the independent variable. The differences in reproduced durations were $M=0.384$ for the short duration, $M=0.357$ for the mid duration, and $M=0.418$ for the long duration. A one-way repeated-measures ANOVA failed to reveal a significant effect of target duration on the coherence order-based difference in reproduced durations $(p=.37)$. Additionally, one-sample $t$ tests suggested that although the positive mean slope parameter of the regression lines relating these difference scores to target durations did not differ significantly from $0(M=0.011$, $p=0.51)$, the intercept $\operatorname{did}(M=0.347, S D=0.404), t(31)=$ $4.86, p<.001$.

Eye-tracking data analyses The change in the number of saccades from encoding to reproduction (henceforth referred to as the saccadic differential) in each target duration condition for each unequal coherence pair is presented in Fig. 4. Visual inspection of Fig. 4 suggests an overlap between the saccadic differential values and normalized reproduced durations, with increasing difference between encoded and reproduced stimulus coherences. This visual overlap suggests a predictive relationship between the saccadic differential and
Fig. 4 Change in the number of saccadic eye movements from training to reproduction in the low coherence given (red line) and low coherence reproduced (blue line) conditions, for the three target durations. Dashed green horizontal line denotes hypothetical zero difference between number of eye movements. Error bars denote standard errors of the mean. (Color figure online)

the reproduced duration. To test this assumption, we have conducted two orthogonal regression analyses separately for the low coherence encoded and low coherence reproduced conditions (solid red and dashed blue lines in Fig. 3, respectively). These analyses were conducted separately for each target duration condition (see Table 4).

Each orthogonal regression analysis tested if the change in the number of eye movements from encoding to reproduction (i.e., the saccadic differential) significantly predicted normalized reproduced durations. Results depicted in Table 4 suggests that in all three target duration conditions, saccadic differential significantly predicted the normalized reproduced durations when there was an increase in coherence from encoding to reproduction (i.e., low coherence encoded condition). On the other hand, saccadic differentials did not predict reproduced durations, when there was a decrease in coherence from encoding to reproduction, in any of the target duration conditions. Taken together, these results suggest that the change in the number of saccadic eye movements from encoding to reproduction is in fact able to index the reproduced duration when there is an accompanying increase in coherence. On the other hand, the predictive power of the saccadic differential disappears when there is a decrease in coherence from encoding to reproduction. Overall, these results point to a conditional predictive relation inherent to the change in saccadic eye movements, with regard to the perceived interval durations.

\section{Discussion}

Our results from all three experiments consistently showed that increasing the coherence of motion from encoding to reproduction resulted in overreproduction of target intervals. This effect was also magnified with increasing difference between motion coherences of two consecutive stimuli and was not mirrored when motion coherence decreased from the encoded to the reproduced stimulus. The difference between encoded and reproduced coherences had virtually no effect on reproduced durations in this latter condition. Additionally, the difference between the number of saccadic eye movements during encoding and reproduction showed a very similar pattern to that seen with reproduced durations (i.e., when higher coherence was reproduced) with regard to unequal coherence pairs. 
a

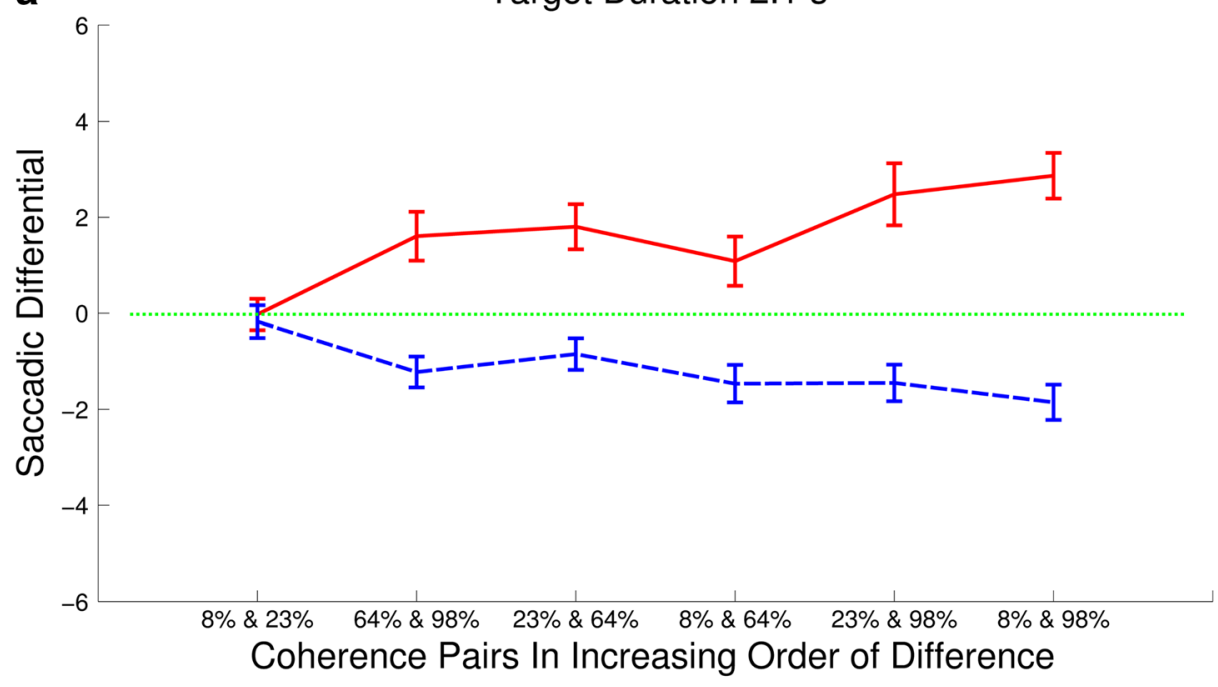

b

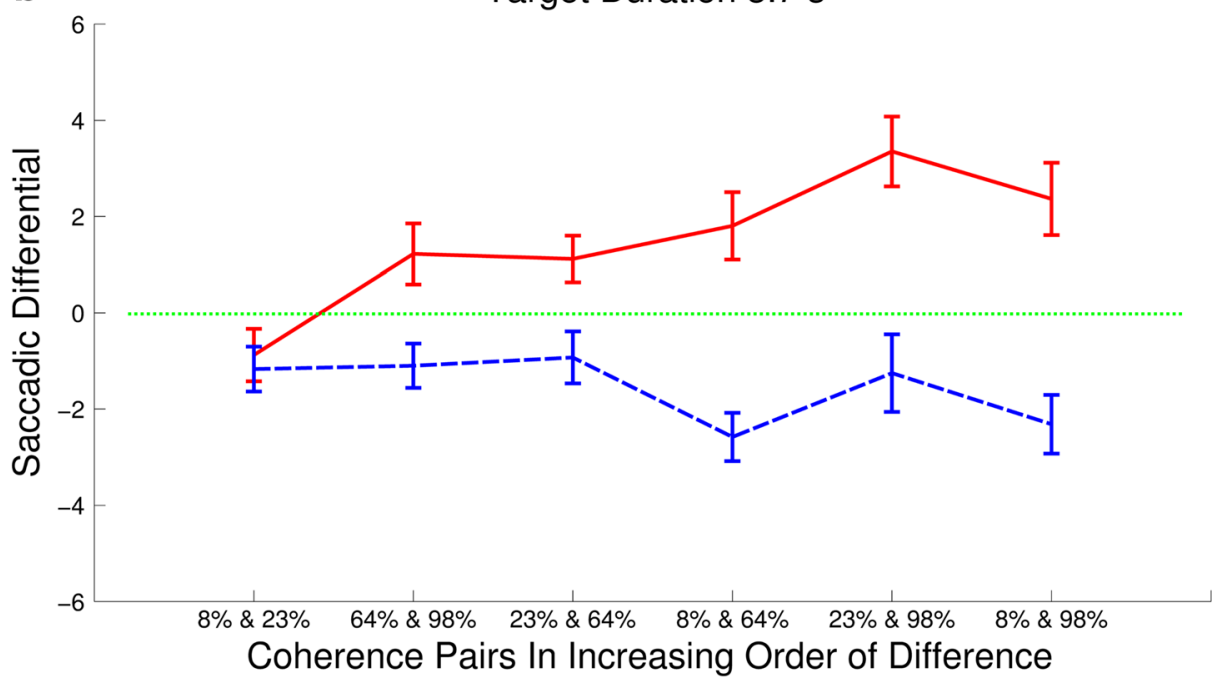

C

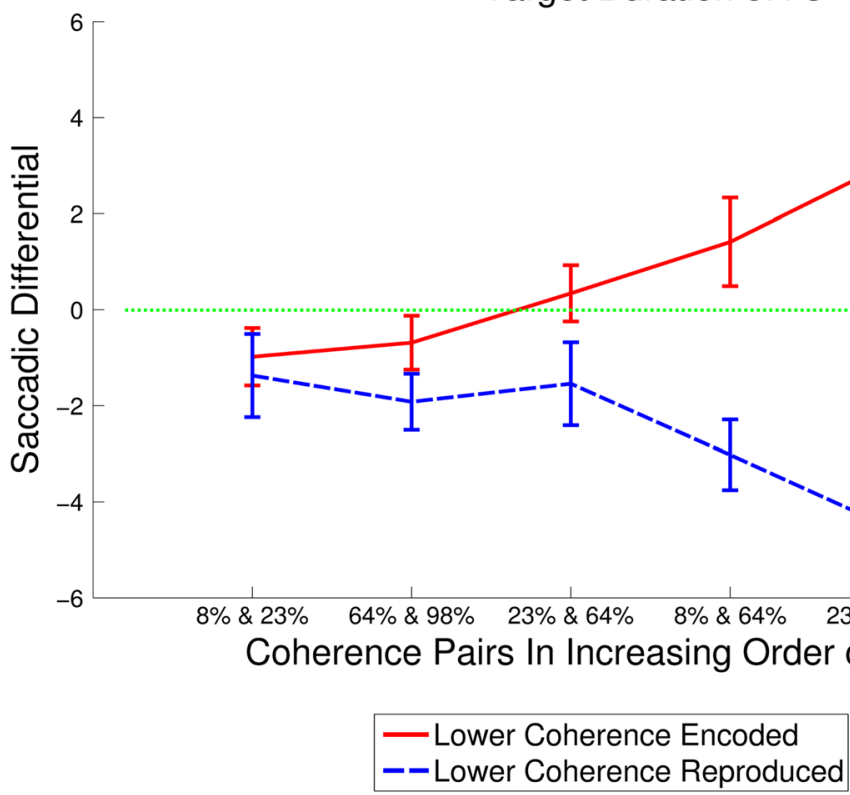


Table 4 Coefficient variables resulting from orthogonal regression analyses for three target duration conditions (2.1, $3.7, \& 5.4$ seconds), with mean normalized reproduced duration as the dependent variable, in Experiment 3

\begin{tabular}{llllll}
\hline & & \multicolumn{4}{l}{ Unstandardized coefficients } \\
\hline Target duration & Experimental condition & $B$ & $S E$ & $Z$ & $p$ \\
2.1 seconds & Lower coherence given & 0.07 & 0.02 & 3.24 & .001 \\
& Lower coherence reproduced & 0.03 & 0.02 & 1.83 & .07 \\
3.7 seconds & Lower coherence given & 0.03 & 0 & 11.02 & .001 \\
& Lower coherence reproduced & 0.02 & 0.01 & 0.97 & .33 \\
& Lower coherence given & 0.02 & 0 & 5.89 & .001 \\
& Lower coherence reproduced & 0 & 0 & 0.99 & .32 \\
\hline
\end{tabular}

Predictor: saccadic differential.
These results are more in line with the predictions of the effect of the motion coherence manipulation on attention to time, compared to its effects on the clock speed. If the effect of our manipulation was on the clock speed, with regard to the cited literature we would expect underreproduction of the target durations when the coherence of the timed stimulus increased from the encoding to reproduction. This prediction is derived under the assumption that, if SNR is assumed to be an indicator of the magnitude of motion in an RDM stimulus, an increase in motion coherence would also increase the clock speed. Such an effect would result in multiplicative (proportional) modulation of the timing behavior. Our data directly oppose this prediction. First and foremost, durations were overreproduced when there was an increase in coherence from encoding to reproduction. Second, the effect of change in motion coherence from encoding to reproduction on interval timing had a prominent additive component (in the opposite direction) in all three experiments.

In terms of the directionality of the observed effects, our findings are more in line with the attentional modulation of perceived durations. This conclusion is based on the finding that higher SNR attracts more attention to sensory stimulus properties (i.e., random motion, speed, directionality) at the expense of attention paid to its temporal properties (Thomas \& Cantor, 1978; Thomas \& Weaver, 1975). This view of attention as being a limited cognitive resource that can be concurrently distributed among different qualities of perceived stimuli (e.g., its temporal and nontemporal properties) and thereby affect temporal perception is well grounded within the timing literature (Brown, 1995; Cantor \& Thomas, 1977; Mattes \& Ulrich, 1998; Thomas \& Cantor, 1978; Thomas \& Weaver, 1975; Tse et al., 2004). There are at least two forms in which attentional modulation can affect the timing behavior. First, the attention-based extension of the scalar timing model (Gibbon et al., 1984) suggests that the clock pulses are gated by a hypothetical switch mechanism to an accumulator, whose "letting through" function is probabilistic (Lejeune, 1998; Penney, 2003) and is thought to be modulated by the amount of attention paid to the temporal properties of an event, where higher attention to time codes for a higher probability of switch closure, and vice versa (Lejeune, 1998; Penney,
Gibbon, \& Meck, 2000; Zakay \& Block, 1995). Second, this switch mechanism is also characterized by alterations in its opening and closing latencies, on the onset and the offset of timing a duration, respectively (Gibbon \& Church 1984; Wearden et al., 1998; Zakay \& Block, 1995). The second mechanism would lead to additive modulation of reproduced durations as it only affects the onset and/or offset of timing, whereas the first mechanism would lead to proportional modulation of reproduced durations as it modulates timing throughout the entire stimulus presentation. In light of these models, our results can partially be explained by assuming that a higher SNR attracted more attention to the nontemporal properties of the timed stimulus and therefore was perceived to be shorter, by acting on either the switch closure probability, the switch opening-closing latencies, or both (see below). However, none of these accounts formulated within a "pacemaker-accumulator" framework predict an asymmetry between identical experimental manipulations made to the encoded and reproduced stimuli (i.e., increase vs. decrease in coherence from encoding to reproduction). This is because the encoding and decoding phases are typically assumed to adhere to identical pacemaker-accumulator contingencies. Consequently, further model specification is necessary to explain the asymmetry in our data, where an increase but not a decrease in SNR led to overreproduction of intervals.

To explain the asymmetrical effects observed in our study, a speculative account of our the attention-based model can be formulated by assuming a one-directional "change detection" or attentional deployment process that is sensitive to an increase but not to a decrease in SNR by the same amount (a precondition for parametric attention-related effects). Within this conjecture, it can be assumed that an increase in SNR from encoding to reproduction might universally attract attention to the stimulus quality that contains the corresponding signal, but at the expense of attention given to its duration. As a result, such a model would predict overreproduction of the target durations when the SNR increases from encoding to reproduction, with no underreproduction when the SNR decreases from encoding to reproduction. Thus, a combination of change detection with the attention-mediated switch mechanism (primarily, latency to closure) can explain 
our findings, including the prominent asymmetries (i.e., the lack of the expected negative slopes in Figs. 2 \& 3, discussed previously).

Similar asymmetries have recently been observed in other studies testing for differences in the discrimination of temporal properties of stimuli using the temporal reproduction methodology (e.g., Cai \& Wang, 2014; Rammsayer \& Verner, 2014). For instance, a looming stimulus leads to time dilation, whereas the opposite effect is not observed for a receding stimulus. (e.g., Van Wassenhove et al., 2008; Van Wassenhove et al., 2011). These findings were hypothesized to result from potential modulations in both clock speed (multiplicative effects) and switch latency (additive effects), and therefore directly relate to and are complemented by the results presented here. Moreover, Raymond and Isaak (1998) have shown that, regardless of the interval between the two stimuli, the coherence threshold of a second RDM stimulus increases when the direction of motion is the same with the first stimulus. This predicts differential degrees of detectability of motion direction of the second stimulus between those cases in which the motion coherence was reduced versus increased from the first to the second stimulus. This finding may also account for the asymmetry in our data. Further studies are necessary to confirm whether motion thresholds vary as such during temporal reproduction.

If an increase in the SNR leads to less attention paid to the temporal aspects throughout the trial (i.e., affect the switch closing probability), then one would expect the magnitude of overreproduction to be proportional to the timed intervals. However, in all experiments, the additive component of the effect was more prominent than the multiplicative component. In fact, there was no reliable multiplicative component of the effect in two out of three experiments. Thus, these unidirectional (low coherence $\rightarrow$ high coherence) additive alterations in timing behavior can be better explained by the effect of the increase in motion coherence on the switch closure latency during the reproduction phase (Block \& Zakay 1996; Gibbon \& Church, 1984; Gibbon et al., 1984; Wearden et al., 1998; Zakay \& Block, 1995, 1996), which would also lead to longer reproductions. Interestingly, this effect increased with the difference between the coherences, suggesting that the switch closure latency also increased as a function of the coherence difference when there was an increased motion coherence. This behavioral observation could be the manifestation of the surprisal/attentional lapse in the case of an increase in SNR, during which the temporal aspects of the event are not processed.

To that end, although the task parameters were identical between Experiments 2 and 3, Experiment 2 revealed a slight multiplicative component to the effect of the increase in motion coherence from encoding to reproduction. Because this effect was in the direction of overreproduction rather than underreproduction of the target intervals, it can also be attributed to the additional effect of the experimental manipulation on switch closure probability. Although participants were instructed not to pay attention to the properties of the RDM stimulus besides its duration (e.g., speed, direction, density), given the close link between attention and the coherence of motion in the highly dynamic RDM stimulus (e.g., Baumann \& Mattingley, 2014; Bolandnazar, Lennarz, Mirpour, \& Bisley, 2015; Liu, Fuller, \& Carrasco, 2006), it is indeed possible that the various levels of coherent motion attracted differential levels of attention paid to the nontemporal properties of the RDM stimulus between encoding and reproduction phases in this experiment. Experiment 3 investigated the potential relationship between the change in the number of eye movements from encoding to reproduction, and the robust behavioral results observed in Experiments 1 and 2 (i.e., reproduced duration). As mentioned previously, saccadic eye movements have been shown to compress (i.e., shorten) perceived durations (Burr et al., 2010; Eagleman, 2005). Our results from Experiment 3 mainly showed that the change in the saccadic eye movements from encoding to reproduction as a function of difference in related motion coherences exhibited a nearly identical pattern to the corresponding changes in the reproduced durations. More specifically, the slope of the lines that relate the saccadic differentials to the lower coherence encoded trial conditions correlated highly with the the slope of the lines that relate the reproduced durations to the identical trial conditions. Additionally this effect was found to hold for all three target durations. On the other hand, there was no relation between eye movements and reproduced durations in conditions where there was a decrease in coherence from encoding to reproduction - which was expected, given the lack of behavioral modulation in this particular condition. These results directly support the "one-directional change detection" variant of the scalar timing model detailed previously, and further complement the behavioral findings observed in Experiments 1 and 2. Given our findings, the change in the number of saccadic eye movements might correlate with the latency to start accumulating temporal information when there is an increase in coherence from encoding to reproduction.

Our analyses of CVs in Experiments 1, 2, and 3 suggest that CVs across durations were not constant. Interestingly, research contesting the constancy of $\mathrm{CVs}$ traditionally shows an increase in $\mathrm{CV}$ with longer/supra-second timed durations (also see Grondin, 2014, for a review); although higher CVs have been found for sub-second intervals as well (see Lewis \& Miall, 2009). Our results in all three experiments contradict these findings, showing that CVs decrease with target durations (see above). Such effects can be accounted for by a generalized form of Weber's law (Getty, 1975; Killeen \& Weiss, 1987), which assumes an additive (i.e., a constant noise) in addition to a scalar source of variability in perceived durations (Ekman, 1959). The additive source of effect 
would be expressed stronger in shorter durations. It is important to note, however, that the design of the current study is not ideal for coming to definitive conclusions regarding the "nonconstancy" of CV.

Finally, overreproduction of all same-coherence pairs in the 2.1-s condition, close-to-target reproduction in the 3.7-s condition, and an underreproduction in the 5.4-s condition in all three experiments is in line with previous research on timing (see Fig. 2). Specifically, these results point at a migration of reproduced durations (a.k.a. memory mixing), possibly because the three durations were randomly assigned to each trial rather than being blocked (i.e., Vierordt's law; Gu \& Meck, 2011; Lejeune \& Wearden, 2009). Additionally, an unexpected result was found in Experiment 1, where reproductions in trials in which the static stimulus was encoded or decoded were longer than the target duration. This result cannot be readily explained by adhering to the effect of the static stimulus exclusively on the allocation of attentional resources (i.e., switch) or on the arousal level (i.e., pacemaker), because opposite behavioral outputs are expected for the two mechanisms. Further, more controlled experiments are necessary to elucidate the differential effect of a static stimulus among dynamic stimuli within the temporal reproduction paradigm.

Our findings point at the modulation of time perception by the complex statistics of the stimulus properties (e.g., increase vs. decrease in SNR), adding to the findings of similar studies that used other stimulus features. Moreover, a robust effect of motion coherence was found in all three experiments, opposing some of the previous work which found no effect of motion coherence on the perception of durations (e.g., Kanai et al., 2006), furthering the discussion on the subject. Based on our findings, specific predictions regarding other functions (e.g., signal detection) can be derived. For instance, the primarily additive nature of the effects of increase in motion coherence suggest that the behavioral effects are driven at the onset of the timed event. Consequently, the detection of other stimulus immediately after the onset (and maybe also at offset) of the timing stimulus might be less likely during the second presentation of the stimulus when there is an increase in SNR. Thus, the asymmetrical effects of our experimental manipulations on timing behavior might also generalize to other domains. Although our results primarily showed an additive effect on timing behavior, we also detected a multiplicative effect in the second experiment. To better estimate the relative contribution of multiplicative and additive effects, future studies can test a wider range of durations. Finally, future studies can test the generality of these findings to other stimulus dimensions, such as size, speed, and brightness, in addition to using eye-tracking methodologies with forced foveal fixation to distinguish between the effect of the perception of motion and the effect of saccadic eye movements induced by that motion.

Acknowledgments This research was supported by a New Agendas for the Study of Time and TÜBA (Turkish Academy of Sciences) GEBIP 2015 grant to F. B. The authors would like to thank Dr. Aylin Küntay for giving access to the eye tracker.

\section{References}

Allan, L. G. (1979). The perception of time. Perception \& Psychophysics, 26(5), 340-354.

Allman, M. J., Teki, S., Griffiths, T. D., \& Meck, W. H. (2014). Properties of the internal clock: First-and second-order principles of subjective time. Annual Review of Psychology, 65, 743-771.

Baumann, O., \& Mattingley, J. B. (2014). Effects of attention and perceptual uncertainty on cerebellar activity during visual motion perception. The Cerebellum, 13(1), 46-54.

Beckmann, J. S., \& Young, M. E. (2009). Stimulus dynamics and temporal discrimination: Implications for pacemakers. Journal of Experimental Psychology: Animal Behavior Processes, 35(4), 525.

Berglund, B., Berglund, U., Ekman, G., \& Frankenhaeuser, M. (1969). The influence of auditory stimulus intensity on apparent duration. Scandinavian Journal of Psychology, 10(1), 21-26.

Beutter, B. R., \& Stone, L. S. (2000). Motion coherence affects human perception and pursuit similarly. Visual Neuroscience, 17(01), 139153.

Block, R. A., \& Zakay, D. (1996). Models of psychological time revisited. Time and Mind, 33, 171-195.

Bolandnazar, Z., Lennarz, B., Mirpour, K., \& Bisley, J. (2015). The role of selective attention during visual search using random dot motion stimuli. Journal of Vision, 15(12), 1366-1366.

Brainard, D. H. (1997). The psychophysics toolbox. Spatial Vision, 10, 433-436.

Brown, S. W. (1995). Time, change, and motion: The effects of stimulus movement on temporal perception. Perception \& Psychophysics, 57, 105-116.

Buhusi, C. V., \& Meck, W. H. (2005). What makes us tick? functional and neural mechanisms of interval timing. Nature Reviews Neuroscience, 6(10), 755-765.

Burr, D. C., Ross, J., Binda, P., \& Morrone, M. C. (2010). Saccades compress space, time and number. Trends in Cognitive Sciences, 14(12), 528-533.

Cantor, N. E., \& Thomas, E. A. (1977). Control of attention in the processing of temporal and spatial information in complex visual patterns. Journal of Experimental Psychology: Human Perception and Performance, 3(2), 243.

Casini, L., \& Macar, F. (1997). Effects of attention manipulation on judgments of duration and of intensity in the visual modality. Memory \& Cognition, 25(6), 812-818.

Cheng, X., \& Penney, T. B. (2015). Modulation of time perception by eye movements. Frontiers in Human Neuroscience, 9. doi:10.3389 /conf.fnhum.2015.219.00049

Droit-Volet, S., Clément, A., \& Wearden, J. (2001). Temporal generalization in 3-to 8-year-old children. Journal of Experimental Child Psychology, 80(3), 271-288.

Eagleman, D. M. (2005). Distortions of time during rapid eye movements. Nature Neuroscience, 8(7), 850-851.

Eagleman, D. M. (2008). Human time perception and its illusions. Current Opinion in Neurobiology, 18(2), 131-136. 
Eagleman, D. M., \& Pariyadath, V. (2009). Is subjective duration a signature of coding efficiency? Philosophical Transactions of the Royal Society, B: Biological Sciences, 364(1525), 1841-1851.

Eisler, H. (1976). Experiments on subjective duration 1868-1975: A collection of power function exponents. Psychological Bulletin, 83, 1154-1171.

Ekman, G. (1959). Weber's law and related functions. The Journal of Psychology, 47, 343-352.

Engbert, R., Sinn, P., Mergenthaler, K., \& Trukenbrod, H. (2015). Microsaccade Toolbox 0.9. Retrieved from http://read.psych.unipotsdam.de/attachments/article/140/MS_Toolbox_R.zip

Getty, D. J. (1975). Discrimination of short temporal intervals: A comparison of two models. Perception \& Psychophysics, 18(1), 1-8.

Gibbon, J. (1977). Scalar expectancy theory and Weber's law in animal timing. Psychological Review, 84(3), 279.

Gibbon, J., \& Church, R. M. (1984). Sources of variance in an information processing theory of timing. In H. L. Roitblat, T. G. Bever, \& H. S. Terrace (Eds.), Animal cognition (pp. 465-488). Hillsdale: Erlbaum.

Gibbon, J., Church, R. M., \& Meck, W. H. (1984). Scalar timing in memory. Annals of the New York Academy of Sciences, 423(1), $52-77$.

Gold, J. I., \& Shadlen, M. N. (2001). Neural computations that underlie decisions about sensory stimuli. Trends in Cognitive Science, 5, 10 16.

Grondin, S. (2010). Timing and time perception: A review of recent behavioral and neuroscience findings and theoretical directions. Attention, Perception, \& Psychophysics, 72(3), 561-582.

Grondin, S. (2014). About the (non) scalar property for time perception. In H. Merchant \& V. de Lafuente (Eds.), Neurobiology of interval timing (pp. 17-32). New York: Springer.

Gu, B. M., \& Meck, W. H. (2011). New perspectives on Vierordt's law: Memory-mixing in ordinal temporal comparison tasks. In A. Vatakis, A. Esposito, M. Giagkou, F. Cummins, \& G. Papadelis (Eds.), Multidisciplinary aspects of time and time perception (pp. 67-78). Berlin: Springer.

Kanai, R., Paffen, C. L., Hogendoorn, H., \& Verstraten, F. A. (2006). Time dilation in dynamic visual display. Journal of Vision, 6(12), 8.

Kaneko, S., \& Murakami, I. (2009). Perceived duration of visual motion increases with speed. Journal of Vision, 9(7), 14.

Killeen, P. R., \& Weiss, N. A. (1987). Optimal timing and the Weber function. Psychological Review, 94(4), 455.

Kleiner, M., Brainard, D., \& Pelli, D. (2007). What's new in Psychtoolbox-3? Perception, 36(ECVP, Abstract Suppl.).

Lappe, M., Awater, H., \& Krekelberg, B. (2000). Postsaccadic visual references generate presaccadic compression of space. Nature, 403(6772), 892-895.

Lejeune, H. (1998). Switching or gating? The attentional challenge in cognitive models of psychological time. Behavioural Processes, 44(2), 127-145.

Lejeune, H., \& Wearden, J. H. (2009). Vierordt's the experimental study of the time sense (1868) and its legacy. European Journal of Cognitive Psychology, 21(6), 941-960.

Leppänen, J. M., Forssman, L., Kaatiala, J., Yrttiaho, S., \& Wass, S. (2014). Widely applicable MATLAB routines for automated analysis of saccadic reaction times. Behavior Research Methods, 47(2), 538-548.

Lewis, P. A., \& Miall, R. C. (2009). The precision of temporal judgement: Milliseconds, many minutes, and beyond. Philosophical Transactions of the Royal Society, B: Biological Sciences, 364(1525), 1897-1905.

Liu, T., Fuller, S., \& Carrasco, M. (2006). Attention alters the appearance of motion coherence. Psychonomic Bulletin \& Review, 13(6), 10911096.
Mattes, S., \& Ulrich, R. (1998). Directed attention prolongs the perceived duration of a brief stimulus. Perception \& Psychophysics, 60(8), $1305-1317$.

Matthews, W. J. (2011). How do changes in speed affect the perception of duration? Journal of Experimental Psychology: Human Perception and Performance, 37(5), 1617.

Merchant, H., Harrington, D. L., \& Meck, W. H. (2013). Neural basis of the perception and estimation of time. Annual Review of Neuroscience, 36, 313-336.

Morrone, M. C., Ross, J., \& Burr, D. (2005). Saccadic eye movements cause compression of time as well as space. Nature Neuroscience, 8(7), 950-954.

Nevalainen, S., \& Sajaniemi, J. (2004). Comparison of three eye tracking devices in psychology of programming research (pp. 151-158). 16th Annual Workshop of the Psychology of Programming Interest Group, Carlow, Ireland. Retrieved from http://www.ppig. org/papers/16th-nevalainen.pdf

Pelli, D. G. (1997). The VideoToolbox software for visual psychophysics: Transforming numbers into movies. Spatial Vision, 10, 437-442.

Penney, T. B. (2003). Modality differences in interval timing: Attention, clock speed, and memory. In W. Meck (Ed.), Functional and neural mechanisms of interval timing (pp. 209-234). Boca Raton: CRC Press.

Penney, T. B., Cheng, X., Leow, Y. L., Bay, A. W. Y., Wu, E., Herbst, S. K., \& Yen, S. C. (2016). Saccades and subjective time in seconds range duration reproduction. Timing \& Time Perception, 4(2), 187206.

Penney, T. B., Gibbon, J., \& Meck, W. H. (2000). Differential effects of auditory and visual signals on clock speed and temporal memory. Journal of Experimental Psychology: Human Perception and Performance, 26(6), 1770.

Rammsayer, T. H., \& Verner, M. (2014). The effect of nontemporal stimulus size on perceived duration as assessed by the method of reproduction. Journal of Vision, 14(5), 1-10.

Raymond, J. E., \& Isaak, M. (1998). Successive episodes produce direction contrast effects in motion perception. Vision Research, 38(4), $579-589$.

Ross, J., Morrone, M. C., \& Burr, D. C. (1997). Compression of visual space before saccades. Nature, 386(6625), 598-601.

Schütz, A. C., Braun, D. I., Movshon, J. A., \& Gegenfurtner, K. R. (2010). Does the noise matter? Effects of different kinematogram types on smooth pursuit eye movements and perception. Journal of Vision, 10(13), 26.

Schütz, A. C., Braun, D. I., \& Gegenfurtner, K. R. (2011). Eye movements and perception: A selective review. Journal of Vision, 11(5), 9.

Suzuki, M., \& Yamazaki, Y. (2010). Distortion of space and time during saccadic eye movements. Intelligent Information Management, 2(02), 90.

Thomas, E. A. C., \& Cantor, N. E. (1978). Interdependence between the processing of temporal and non-temporal information. In J. Requin (Ed.), Attention and performance VII (pp. 43-62). Hillsdale: Erlbaum.

Thomas, E. A., \& Weaver, W. B. (1975). Cognitive processing and time perception. Perception \& Psychophysics, 17(4), 363-367.

Tomassini, A., Gori, M., Burr, D., Sandini, G., \& Morrone, M. C. (2011). Perceived duration of visual and tactile stimuli depends on perceived speed. Frontiers in Integrative Neuroscience, 5. doi:10.3389 /fnint.2011.00051

Tse, P. U., Intriligator, J., Rivest, J., \& Cavanagh, P. (2004). Attention and the subjective expansion of time. Attention, Perception, \& Psychophysics, 66, 1171-1189.

Van Wassenhove, V., Buonomano, D. V., Shimojo, S., \& Shams, L. (2008). Distortions of subjective time perception within and across senses. PLoS ONE, 3(1), e1437. 
Van Wassenhove, V., Wittmann, M., Craig, A. D., \& Paulus, M. P. (2011). Psychological and neural mechanisms of subjective time dilation. Frontiers in Neuroscience, 5, 56.

Wearden, J. H., Edwards, H., Fakhri, M., \& Percival, A. (1998). Why "'sounds are judged longer than lights"': Application of a model of the internal clock in humans. The Quarterly Journal of Experimental Psychology: Section B, 51(2), 97-120.

Xuan, B., Zhang, D., He, S., \& Chen, X. (2007). Larger stimuli are judged to last longer. Journal of Vision, 7(10), 2.

Yamamoto, K., \& Miura, K. (2016). Effect of motion coherence on time perception relates to perceived speed. Vision Research, 123, 56-62.
Yarrow, K., Haggard, P., \& Rothwell, J. C. (2004). Action, arousal, and subjective time. Consciousness and Cognition, 13(2), 373-390.

Zakay, D., \& Block, R. A. (1995). An attentional gate model of prospective time estimation. In V. De Keyser, G. d'Ydewalle, A. Vandierendonck, \& G. d'Ydewalle (Eds.), Time and the dynamic control of behavior (pp. 167-178). Seattle: Hogrefe \& Huber.

Zakay, D., \& Block, R. A. (1996). The role of attention in time estimation processes. Advances in Psychology, 115, 143-164.

Zelkind, I. (1973). Factors in time estimation and a case for the internal clock. The Journal of General Psychology, 88(2), 295-301. 